\title{
A Taxonomic Account of Hover Flies (Insecta: Diptera: Syrphidae) with 4 New Records from Cold Dry Zones of Himachal Pradesh, India.
}

\author{
Jayita Sengupta $^{1 \star}$, Atanu Naskar ${ }^{1}$, Aniruddha Maity $^{2}$, Sumit Homechaudhuri ${ }^{3}$, Dhriti Banerjee ${ }^{4}$ \\ ${ }^{1}$ Senior Zoological Assistant, Diptera Section, Zoological Survey of India, Kolkata, India \\ ${ }^{2}$ Senior Research Fellow, Diptera Section, Zoological Survey of India, Kolkata, India. \\ ${ }^{3}$ Professor, Department of Zoology, University of Calcutta, Kolkata, India. \\ ${ }^{4}$ Scientist-D, Diptera Section, Zoological Survey of India, Kolkata, India.
}

Correspondence E-mail : jayitasengupta9@gmail.com; atanudiptera@gmail.com; armzool2007@gmail.com; sumithomechaudhuri@gmail.com,dhritibanerjee@gmail.com.

\begin{abstract}
Eighteen (18) species of hover flies (Insecta:Diptera:Syrphidae) under 14 genera and 2 subfamilies has been reported from the Cold dry zone of Himachal Pradesh which includes Kinnaur, Lahul \& Spiti and Pangi range of Chamba districs. 4 species namely Chrysotoxum violaceum Brunetti, 1923, Sphiximorpha triangulifera (Brunetti, 1913), Mallota (Mallota) orientalis (Wiedemann, 1824), Mallota (Mallota) varicolor(Walker, 1856) has been reported from the first time from this cold and dry zone zone as well as from the state of Himachal Pradesh. Their taxonomic keys and detail diagnosis of the reported species has been discussed along with the distributional pattern of these species along the cold dry zone of Himachal Pradesh.
\end{abstract}

Keywords: Taxonomy, Hover flies, Syrphidae, New Record, cold dry zone, Himachal Pradesh.

\section{Introduction}

The flies of family Syrphidae (Insecta: Diptera: Brachycera) is commonly known as Hover flies or flower flies. Hover flies are distributed worldwide largely with 6,000 known species placed in more than 300 distinctive group. (Pape \& Thomson, 2018).Almost all adult syrphids visit flower for pollen and nectar while larval stages exhibited a very diverse array of feeding modes with complex morphological and behavioural adaptations. Hoverflies are usually variable in body size ranging from small to large, slender to robust in shape and size. The main identifying features lies in the presence of spurious vein or false vein between the $3^{\text {rd }}$ and $4^{\text {th }}$ vein of the wing. Syrphid flies cabn also be distinguished by yellow and orange markings on head, thorax and abdomen. Many of this pollinator flies are excellent mimics of aculeate hymenoptera.

Syrphidae is one of the most common high altitude insect (Mani, 1968). Syrphis flies act as predominant pollinator especially at an elevational range of 1500 meter and above whereas bees and beetles decreased rapidly in such elevational range. Because climate change is expected to strongly affect the mountain eco systems, it has become urgent to develop a better knowledge of the pollinators involved in pollination in such eco system. Our present study area thus expanding through an elevational range of from 2,200 $\mathrm{mt}(7,218$ feet) to the highest point of greater Himalaya in this state of Himachal 
Pradesh. This zone is more commonly known as the Cold \& Dry Zone.

Phytophagous flower flies are very important group of insects because of their two fold services to the ecosystem. Larvae are important natural enemies of herbivorous arthropod while adults play dominant role in pollination (Tooker et. al., 2006, Ghahari et al.2008). Their importance as predator is equal to that of parasitoid, lady bird beetles, pathogenic fungi. (Ankersmit et al.1986).

Pollination and biological control are one of the most important ecosystem service rendered by insects to human being. (Potts et al., 2006). Any loss in biodiversity is a matter of public concern but losses of pollinators are troubling because it will potentially affect the reproduction status of food crops and other plants of agricultural, medicinal importance. Thereby loss of pollinators rise a question while considering our future food security. Therefore taxonomic studies of this pollinators for proper identification is quite necessary. In the present study a brief synopsis on the taxonomy of the family is given along with its distributional pattern in the cold dry zone of Himachal Pradesh.

\section{Materials and Methods}

\section{A. Study area:}

According to the Department of Agriculture, Himachal Pradesh the agro climatic condition has divided the whole state of Himachal Pradesh into 4 zones namely Shivalik hill zones, mid hill zones, high hill zone and cold and dry zone. Our present work is associated with the study areas from cold and dry zone of Himachal Pradesh. Our current study area includes the whole of Kinnaur, Lahul \& Spiti and Pangi range of Chamba districts. Elevation of this zone ranges in approximately from 2,200 $\mathrm{mt}(7,218$ feet) to the highest point of greater Himalaya in this state. This zone comprises about $8 \%$ of total geographical area of the State and $2 \%$ of the total cultivated area of the total state. The very low temperature range and more less rainfall has reduced the percentile of agricultural contribution from this agro climatic zone of Himachal Pradesh. Along the eastern boundary of this zone, rivers are abundant, main rivers of this range are
Satluj, Beas and Spiti. The cold desert of Spiti valley is situated in this Zone. Geographically this zone of Himachal Pradesh is very close to Tibet and Chaina border. Zanskar range on the eastern side of this zone separates Kinnaur and parts of Lahul \& Spiti from Tibet. Normal rainfall is remarkably low in this zone (50-100 mm) but snow fall is very heavy resulting in the formation of cold dry zone in this upper elevation area.

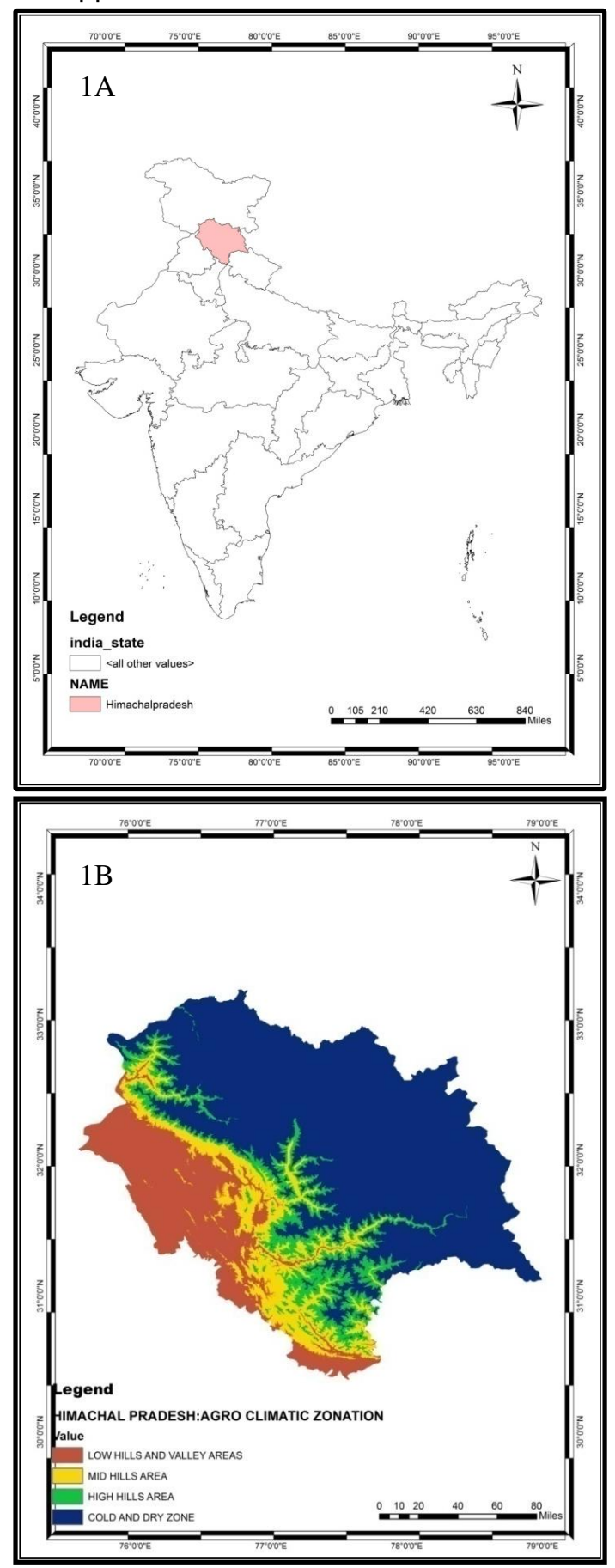

Figure 1A-1B: 3D Map showing 1A: State of Himachal Pradesh, 1B: Agro climatic zonation from the state of Himachal Pradesh. 


\section{B. Collection method:}

For the purpose of collection of hover flies, a 2 year long survey (2017-2018) has been conducted in this cold dry zone of Himachal Pradesh. Hoverflies were collected from the field during day time by using insect sweep nets, different type of traps like malaise trap, pan trap and UV light traps were used for collecting syrphid fauna. The collected samples are narcotized by using ethyl acetate and stored for further study in insect envelopes in the field. The specimens were later carried back to the laboratory, mounted on insect pins, labelled using the collection site information and stored in insect cabinets for further identification.

C. Identification of specimens:

Identification of the adults was done by following the keys of Miranda (2013),

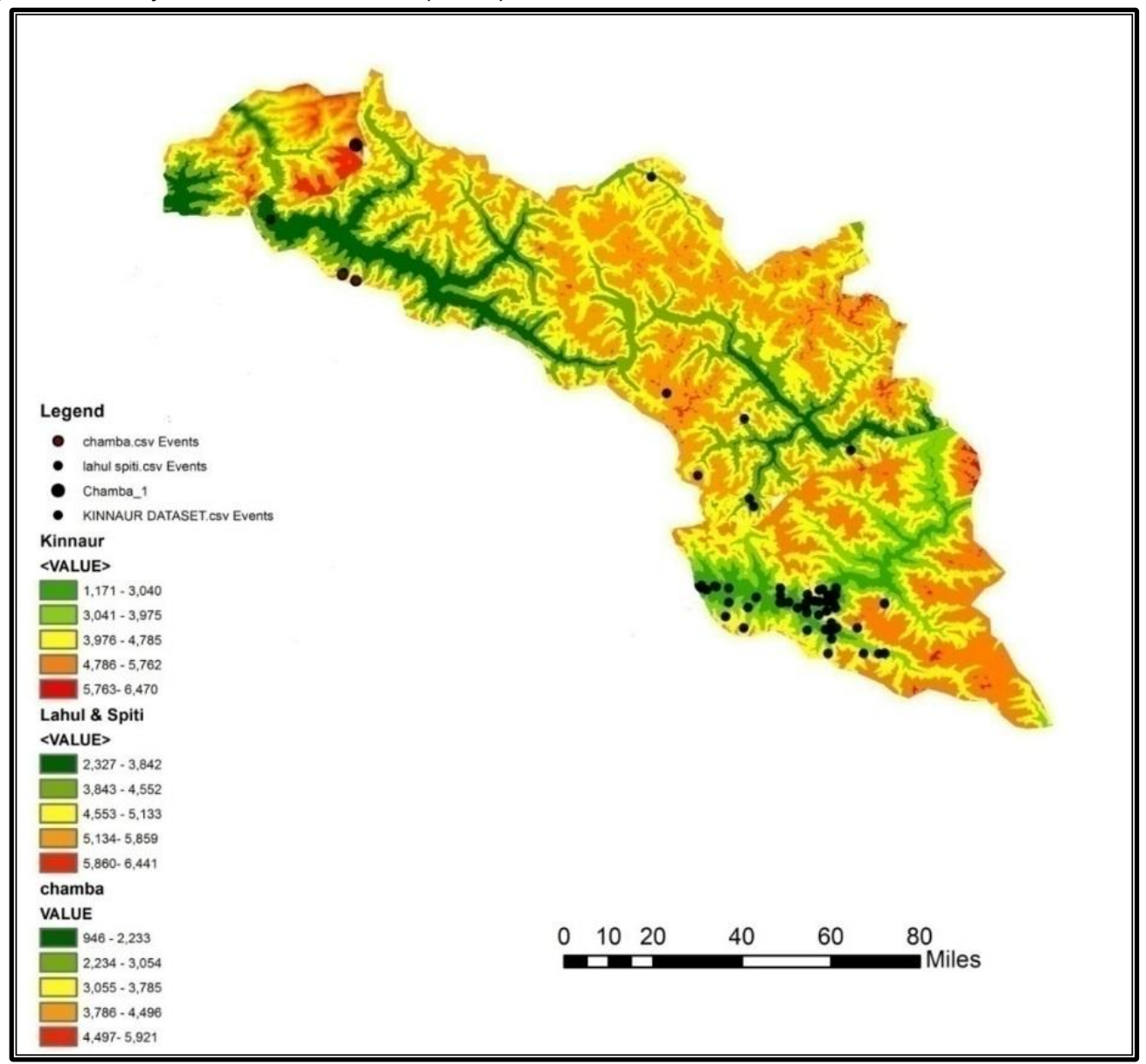

Figure 2: 3D Map showing species richness from Cold and Dry zone from the state of Himachal Pradesh.
Vockeroth (1992) and Brunetti (1923) keeping in mind the recent nomenclatural changes (Pape and Thompson, 2018.Same has been used to construct taxonomic keys. All terminology while describing morphology has followed the recent pattern (McAlpine et al. 1987).All the identified specimens were deposited in the designated repository of National Zoological Collection, Diptera section, Zoological Survey of India, Kolkata.

D. Technical procedure:

The 3D map of study area used here is generated by using ARC GIS software version 10.1. The photograph of habitus were taken by using Leica Microscope M205A, where $0.32 x$ Acro lense was used for habitus photography. 
Results

Altogether 18 species of hoverflies under 14 genera and 2 sub families have been reported from our study area. Among which 4 species namely Chrysotoxum violaceum Brunetti, 1923, Sphiximorpha triangulifera (Brunetti, 1913), Mallota (Mallota) orientalis (Wiedemann, 1824), Mallota (Mallota) varicolor (Walker, 1856) has been reported from the first time from this cold and dry zone as well as from the state of Himachal Pradesh. Detailed systematic account along with taxonomic key has been discussed. Distribution pattern of all syrphid species has been discussed in detail.

\section{List of Taxa}

Family Syrphidae (New Records from the state of Himachal Pradesh has been demarcated with asterisk)

\section{Subfamily Syrphinae}

Tribe Syrphini

I. Genus Episyrphus Matsumura \& Adachi, 1917

Subgenus Episyrphus Matsumura \& Adachi, 1917

\section{Episyrphus (Episyrphus) balteatus (De Geer, 1776)}

II. Genus Scaeva Fabricius, 1805
2. Scaeva latimaculata (Brunetti, 1923)

3. Scaeva pyrastri (Linnaeus, 1758)**

III. Genus Sphaerophoria Lepeletier \& Serville, 1828

Subgenus Sphaerophoria Wiedemann, 1830

\section{Sphaerophoria \\ (Sphaerophoriascripta) indiana Bigot, 1884}

IV. Genus Syrphus Fabricius, 1775

Subgenus Syrphus Fabricius, 1775

\section{Syrphus (Syrphus) torvus Osten Sacken, $1875^{\star \star}$}

V. Genus Chrysotoxum Meigen, 1800
6. Chrysotoxum violaceum Brunetti, $1923^{\star *}$

Tribe Bacchini

VI. Genus Melanostoma Schiner, 1860

7. Melanostoma orientale (Wiedemann, 1824)

Tribe Paragini

VII. Genus Paragus Latreille, 1804 Subgenus Paragus Latreille, 1804

8. Paragus (Paragus) bicolor (Fabricius, 1794)

Subfamily Eristalinae

Tribe Volucellini

VIII. Genus Volucella Geoffroy, 1762

9. Volucella ruficauda Brunetti, 1907

IX. Genus Sphiximorpha Rondani, 1850

10. Sphiximorpha triangulifera (Brunetti, 1913) $^{\star \star}$

Tribe Eristalini

X. Genus Eristalinus Rondani, 1845

Subgenus Eristalodes Mik, 1897

11. Eristalinus (Eristalodes) paria (Bigot, 1880)

XI. Genus Eristalis Latreille, 1804

Subgenus Eoseristalis Kanervo, 1938

12. Eristalis (Eoseristalis) cerealis Fabricius, 1805

13. Eristalis (Eoseristalis) himalayensis Brunetti, 1908

Subgenus Eristalis Latreille, 1804

14. Eristalis (Eristalis) tenax (Linnaeus, 1758)

XII. Genus Phytomia Guerin-Meneville, 1833

Subgenus Dolichomerus Macquart, 1850

15. Phytomia (Dolichomerus) crassa (Fabricius, 1787)

XIII. Genus Mallota Meigen, 1822

Subgenus Mallota Meigen, 1822 
16. Mallota (Mallota) orientalis (Wiedemann, 1824) ${ }^{\star \star}$

17. Mallota (Mallota) varicolor (Walker, 1856) $)^{\star \star}$

XIV. Genus Syritta Lepeletier \& Serville, 1828

18. Syritta pipiens (Linnaeus, 1758)

Key to sub families, tribe, genera and species of hover flies from the cold dry zone of Himachal Pradesh.

\section{Systematic account}

Key to sub families

1. Bare post pronotum, head strongly concave at posterior end, in male $5^{\text {th }}$ abdominal tergite is visible from dorsal surface.

Syrphinae

- Few sparse hairs on post pronotum, head weakly concave at posterior end, in male $5^{\text {th }}$ abdominal tergite is not visible from dorsal

surface.

Eristalinae

Sub family Syrphinae

Key to tribe

1. Posterior margin of scutellum strongly denticulate. Paragini

Posterior margin of scutellum not denticulate. .2

2. Shape of abdomen oval or equilateral, mesopleural haring usually not present. Syrphini

- Shape of abdomen distinctly petiolate, mesopleuron with erect coarse hairs on postero dorsal corner. Bacchini

Key to genera of tribe Syrphini

1. Strongly convex abdomen, elongated antennae usually longer than head........Chrysotoxum Meigen, 1800

- Shape of abdomen variable, short antennae usually shorter than head.................................................

2. Lower lobe of calypter contains long ochre yellow hairs. Syrphus, Fabricius, 1775
- Lower lobe of calypter contains microscopic ground color hairs...................................................

3. Entirely black face and scutellum..................................Melan ostoma,Schiner, 1860

- Yellow or partly yellowish face and scutellum............................................ 4

4. Sharply defined lateral or sub lateral stripes present at scutum....................Sphaerophoria, Lepeletier \& Serville, 1828

- Poorly defined lateral stripe present at scutum.

5. Minutely punctated tergites............Paragus Latreille, 1804

- Tergites without any punctuation......................................... 6

6. Apical one third half of wing membrane is densely trichose. Episyrphus Matsumura \& Adachi, 1917

- Whole surface of wing membrane covered with very sparse and scattered

microtrichia... Scaeva

Fabricius, 1805

Key to species of genera Scaeva Fabricius, 1805

1. Abdominal tergites with white colored comma like structures extending towards the middle.

pyrastri (Linnaeus, 1758)

Abdominal tergites with yellow colored comma like structures extending towards the middle.....latimaculata (Brunetti, 1923)

\section{Sub family Eristalinae}

Key to tribe

1. Anterior part of mesopleuron usually bare, anterior basal patch of setulae on hind femur.

Eristalini

Anterior portion of meso pleuron usually pilose, no patch of setulae on hind femur. 
2. Wing usually with perpendicular anterior cross vein before middle of discal cell, hind femur with basal patch of setulae.

Volucellini

- Wing usually with slanted anterior cross vein beyond middle of discal cell, hind femur without any patch of setulae...................... 3

3. $3^{\text {rd }}$ antennal segment tapered to a point, always with a terminal style...............Ceriodini

- $\quad 3^{\text {rd }}$ antennal segment elongated and with a terminal

style.

Milesini

Key to genera of tribe Eristalini

1. Scutellum abnormally wide, frons with wrinkled space above anntenal segment..............Phytomia

Guerin-Meneville, 1833

- Scutellum of normal shape, no wrinkled space is there above antennal segment. 2

2. Scuttum unstriped, abdomen dark without pale markings,wing membrane's basal one third portion usually bare. Mallota Meigen, 1822

- Scutum usually with longitudinal stripes, abdomen with pale markings, wing membrane entirely covered with micro trichia. .3

3. Katepimeron and metepisternum wholly bare.........Eristalis Latreille, 1804

- Katepimeron haired and metepisternum partly haired Eristalinus Rondani, 1845

Key to subgenera of genera Eristalis

XV.

1. Posterior half of anepimeron bare....Eoseristalis Kanervo, 1938

- Posterior half of anepimeron partlty haired....................Eristalis Latreille, 1804

Key to species of genera Eristalis
1. Arista partly or wholly plumose..............2 - Arista completely bare.........tenax (Linnaeus, 1758)

2. Abdominal tergites all black except yellowish margins himalayensis Brunetti, 1908

Presence of yellowish spots or markings on abdominal tergites......cerealis Fabricius, 1805

Key to species of genera Mallota

1. Thorax pale yellow in color, usually smaller species.......orientalis (Wiedemann, 1824)

- Anterior portion of Thorax dark brown in color, usually larger species...........varicolor (Walker, 1856)

Key to genera of tribe Volucellini

1. Frontal prominence absent or much shorter than scape. Long haired flies, mimics bumble bee.......... Volucella Geoffroy, 1762

- Frontal prominence at least as long as scape. Flies without dense long hairs on body, never mimics bumble bee............Sphiximorpha Rondani, 1850

\section{Subfamily Syrphinae}

Diagnosis: Face without distinct eye margin, or such a zone only present on lower part of the face. Humeri bare. The majority of species of this subfamily have a distinct colour pattern of spots or bars on abdomen.

\section{Tribe Syrphini}

Diagnosis: Yellow scutellum distinguishes this tribe from others. Face in most species is partially or completely yellow. $3^{\text {rd }}$ antennal segment usually more compact or rarely elongated.

I. Genus Episyrphus Matsumura \& Adachi, 1917

Type species: Musca balteata De Geer

Diagnosis:

Subgenus Episyrphus Matsumura \& Adachi, 1917 


\section{Episyrphus (Episyrphus) balteatus (De Geer, 1776)}

1776. De Geer, Mem. pour. serv. Hist. Ins.6: 116

1924. Syrphus signatus Abreu,Dipt. Tijdsr. Ent.10:144

\section{Type locality: Sweden}

Material examined: $4 \hat{\partial} \hat{\partial}$, Bhowen, Chamba district, $2200 \mathrm{mt}$; $32^{\circ} 29^{\prime} 55.60^{\prime \prime} \mathrm{N}$, 76 5'27.40"E, 15.iv.17, coll: J.Sengupta, 4우우, 1스, Hadsar, Chamba district, 3100 $\mathrm{mt}, \quad 32^{\circ} 27^{\prime} 22.08^{\prime \prime} \mathrm{N}, \quad 76^{\circ} 36^{\prime} 53.16^{\prime \prime} \mathrm{E}$, 16.iv.17, coll: J.Sengupta,1 1 , Bhabanagar, Kinnaur district, $1520 \mathrm{mt}$; 31 $333^{\prime} 51^{\prime \prime N}, \quad 77^{\circ} 55^{\prime} 44^{\prime \prime E}$, 12.iv.18, coll: J.Sengupta, $4 \hat{\delta} \hat{\jmath}, \quad 5$ 웅, Recong peo, Kinnaur district, $1610 \mathrm{mt}$; $31^{\circ} 30^{\prime} 58^{\prime \prime} \mathrm{N}$, $78^{\circ} 5$ '47"E,12.iv.18, coll: J.Sengupta, 1 , Kinnaur hill side, Kinnaur district, $1600 \mathrm{mt}$, $31^{\circ} 33^{\prime} 51 " \mathrm{~N}, \quad 77^{\circ} 50$ '25"E, $12 . i v .18$ coll: J.Sengupta, 2 + , Bhujund, Kinnaur district, $3028 \mathrm{mt}, 32^{\circ} 45^{\prime} 46.90^{\prime \prime} \mathrm{N}$, 76²6'15.16"E, 12.iv.18, coll: J.Sengupta, $4 \hat{\jmath} \widehat{\alpha}, 5$ 우 $q$,Sangla,Kinnaurdistri ct, $1980 \mathrm{mt}, 31^{\circ} 28^{\prime} 32 " \mathrm{~N}, 78^{\circ} 11^{\prime 2} 2 " \mathrm{E}, 13$.iv.18,c oll:J.Sengupta1,1 $\delta^{\lambda}$, Kuppa, Kinnaur district, $1990 \mathrm{mt}, 31^{\circ} 28^{\prime} 56^{\prime \prime} \mathrm{N}, 78^{\circ} 11^{\prime} 4^{\prime \prime} \mathrm{E}$,

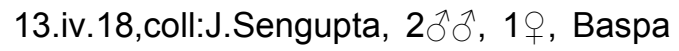
valley garden, Kinnaur district, $2680 \mathrm{mt}$, $31^{\circ} 25^{\prime} 34^{\prime \prime} \mathrm{N}$, $78^{\circ} 16^{\prime} 5^{\prime \prime} \mathrm{E}$, 13.iv.18,coll:J.Sengupta,19, Kugti, Lahul \& Spiti district, $4630 \mathrm{mt}, 32^{\circ} 28^{\prime} 42.58^{\prime \prime} \mathrm{N}$, 7653'5.95"E, 16.iv.18, coll: J.Sengupta, $1{ }^{\AA}$, Gemur, Lahul \& Spiti district, $4827 \mathrm{mt}$, 32'36'21.32"N, $\quad 77^{\circ} 10^{\prime} 11.76 " \mathrm{E}$, 16.iv.18,coll: J. Sengupta, $2 \hat{\jmath}$, Batal, Lahul \& Spiti district, $4737 \mathrm{mt}$, 32²0'35.29"N, 7742'26.63"E, 16.iv.18, coll: J.Sengupta.

Diagnosis: Double black bands on $3^{\text {rd }}$ and $4^{\text {th }}$ abdominal tergites, strength of this marking is very variable. Anterior black band can be reduced to two black dashes while the dark forms tend to have the lateral margins darkened. Narrow median grey stripe anteriorly flanked by another grey stripe on either side. All these stripes together fused into an extensive grey area at the back of thoracic dorsum. Wing length $6-10.25 \mathrm{~mm}$.
Distribution: India: Widely distributed through all the states of India.

Distribution: elsewhere: Australasian Region (Australia), Oriental Region (Widely distributed), Palearctic Region (England).

Remarks: This species is very common throughout the whole of the East in both plain land and hill region during summer. The range of this widely distributed species includes Europe, North Africa, Asia to Japan.

II. Genus Scaeva Fabricius,

Type species: Musca pyrastri Linnaeus.

Diagnosis: Frons distinctly inflated. Face with a weak stripe not extending above knob. Tergite 3 and 4 entirely black with hairs on lateral margins.

\section{Scaeva latimaculata (Brunetti, 1923)}

1923. Lasiopticus latimaculata Brunetti, Fauna. Br. India. Dipt.3:68

1975. Scaeva montana Violovitsh, Ent. Obozr.54:173

Type locality: Allahabad, Peshawar, Ferozepore and Abu, India

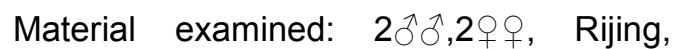
Kinnaur district, $3130 \mathrm{mt}, 31^{\circ} 38^{\prime} 55.27^{\prime \prime} \mathrm{N}$ 78²4'20.72"E, 16.iv.18, coll: J.Sengupta,

Diagnosis: Frons distinctly inflated, maxilla black to brownish orange in colour range, with orange coloured arista. Thorax aeneous black in colour with translucent yellowish scutellum. Abdomen glossy black with 3 pairs of broad orange spots. Abdominal tergites covered with ground coloured pubescence.

Distribution: India: Himachal Pradesh, Delhi, Jammu \& Kashmir, Punjab, Rajasthan, Uttar Pradesh, Uttarakhand, West Bengal.

Distribution: elsewhere: Oriental region (Pakistan)

Remarks: This species is demarcated by its broad and oval abdominal spots. 


\section{Scaeva pyrastri (Linnaeus, 1758) ${ }^{\star \star}$}

1758. Musca pyrastri Linnaeus, Syst. Nat.1:594

1884. Syrphus flavoscutellatus Girschner, Wien. ent. Ztg.3 (7): 197

Type locality: Sweden

Material examined: $2 \hat{\delta}$, Kalpa agricultural field, Kinnaur district, $2380 \mathrm{mt}$, 31'32'59"N,78¹5 '6"E, 15.iv.18, coll: J.Sengupta, 1\%, Kalpa garden area, Kinnaur district, $2790 \mathrm{mt}$, 31'32'23"N,78¹5 '9"E, 15.iv.18, coll: J.Sengupta, $2 \circ+$, Chitkul valley, Kinnaur district, $3350 \mathrm{mt}, \quad 31^{\circ} 21^{\prime} 2 " \mathrm{~N}, 78^{\circ} 25^{\prime} 5 " \mathrm{E}$, 15.iv.18, coll: J.Sengupta, $29+$, Chitkul forest, Kinnaur district, $3360 \mathrm{mt}$, 31'20'58"N,78²6'14"E, 15.iv.18, coll: J.Sengupta, $2 \hat{\delta} \widehat{\partial}$, Baspa Valley, Kinnaur district, $3400 \mathrm{mt}, 31^{\circ} 21^{\prime} 2 " \mathrm{~N}, 78^{\circ} 26^{\prime} 10^{\prime \prime} \mathrm{E}$, 15.iv.18, coll: J.Sengupta, $3 \delta^{\widehat{\lambda}}, 2$ 웅, Rakcham valley, Kinnaur district, $3410 \mathrm{mt}$, 31'21'3"N,78²6'12"E, 15.iv.18, coll: J.Sengupta,

Diagnosis: Relatively large conspicuous hoverfly, abdominal tergites have distinctive lunules. Tergite 3 and 4 with hooked bars of almost equal width at each end and with outer end not reaching as far forward as inner end. Markings on abdominal tergites are whitish in colour. $\mathrm{R}_{4+5}$ vein bowed, microtrichia are extensively absent.

Distribution: India: Himachal Pradesh, Jammu \& Kashmir, Punjab, Uttarakhand.

Distribution: elsewhere: Palearctic Region (Germany), Nearctic Region (Alaska, California, Mexico,)

Remarks: This species is recorded newly from the state of Himachal Pradesh. This species is easily distinguishable by the presence of whitish lunules on dorsal surface of abdominal tergites.

\section{Genus Sphaerophoria Lepeletier \& Serville, 1828}

Type species: Musca scripta Linnaeus.

Diagnosis: Unmarginated abdominal tergites, ventral scutellar finge usually absent, in make fly, terminalia extremely large.
Subgenus Sphaerophoria Wiedemann, 1830

\section{Sphaerophoria}

(Sphaerophoriascripta)indiana Bigot, 1884

1884. Sphaerophoria indiana Bigot, Annls. Soc. ent. Fr. (6) 4: 99

1916. Melithreptus diminutus Matsumura, Ent. Mag. Kyoto.2: 27

Type locality: Indes.

Material examined: $3 \hat{\partial} \hat{\jmath}$, Hadsar, Chamba district, $\quad 3100 \mathrm{mt}, \quad 32^{\circ} 27^{\prime} 22.08^{\prime \prime} \mathrm{N}$, 76³6'53.16"E, 16.iv.17, coll: J.Sengupta, $7 \AA \hat{\partial}$, Rampur power project, Kinnaur district, $970 \mathrm{mt}, 31^{\circ} 23^{\prime} 38^{\prime \prime} \mathrm{N}, 7^{\circ} 36^{\prime} 2 " \mathrm{E}$, 14.iv.18, coll: J.Sengupta, $6 ㅇ ㅜ, 3{ }^{\lambda} \delta^{\lambda}, K u p p a$, Kinnaur district, 1990 $\mathrm{mt}, \quad 31^{\circ} 28^{\prime} 56 " \mathrm{~N}, \quad 78^{\circ} 11^{\prime} 4 " \mathrm{E}$, 13.iv.18,coll:J.Sengupta, $\quad 2 \delta^{\lambda}$, 2 우 ,Bhujund, Kinnaur district, $3028 \mathrm{mt}$, $32^{\circ} 45^{\prime} 46.90 " \mathrm{~N}, \quad 76^{\circ} 26^{\prime} 15.16 " \mathrm{E}, \quad 12 . i v .18$, coll: J.Sengupta, 1, Kugti, Lahul \& Spiti district, $4630 \mathrm{mt}, \quad 32^{\circ} 28^{\prime} 42.58^{\prime \prime} \mathrm{N}$, 7653'5.95"E, 16.iv.18, coll: J.Sengupta, $1{ }^{\lambda}$, Gemur, Lahul \& Spiti district, $4827 \mathrm{mt}$, 32०36'21.32"N, $\quad 77^{\circ} 10^{\prime} 11.76 " \mathrm{E}$, 20.iv.18,coll: J. Sengupta, $2 \widehat{\partial}$, Batal, Lahul \& Spiti district, $4737 \mathrm{mt}$, 32²0'35.29"N, 7742'26.63"E, 19.iv.18, coll: J.Sengupta, $4 \circ q$, Kugti, Lahul \& Spiti district, $4630 \mathrm{mt}, \quad 32^{\circ} 28^{\prime} 42.58^{\prime \prime} \mathrm{N}$, 7653'5.95"E, 19.iv.18, coll: J.Sengupta,

Diagnosis: Thoracic disc with complete yellow side stripes. Abdomen extends well beyond wing tips. Surstylus very broad. Inner process broad at base, abruptly slender to apex.

Distribution: India: Himachal Pradesh, Arunachal Pradesh, Assam, Bihar, Delhi, Jammu \& Kashmir, Karnataka, Kerala, Maharashtra, Manipur, Meghalaya, Mizoram, Punjab, Sikkim, Tripura, Uttarakhand, Uttar Pradesh, West Bengal.

Distribution: elsewhere: Oriental Region (Bhutan, Myanmar, Nepal, Pakistan, Sri Lanka), Palaearctic Region (China, Japan, Korea)

Remarks: This is a very common Indian species distributed widely throughout the 
Country occurring mostly from December to May month.

$$
\begin{aligned}
& \text { Genus Syrphus Fabricius, } \\
& 1775
\end{aligned}
$$

Type species: Musca rebesii Linnaeus.

Diagnosis: Frons not conspicuously produced, face with central bump, abdomen slender in appearance, marginal cell of wing remain open, lower lobe of calypter with many long coarse yellow hairs.

\section{Subgenus Syrphus Fabricius, 1775}

\section{Syrphus (Syrphus) torvus Osten Sacken, 1875}

1875. Syrphus torvus Osten Sacken, Pros. Boston Soc. Nat. Hist.18:139

1940. Syrphus discretus Szilady, Ann. Mus. Nat. Hung. (Zool). 33:63

Type locality: Canada; Colorado, N.H., R.I., USA.

Material examined: $2 q q$, Bhemkali temple side garden, Kinnaur district, $2130 \mathrm{mt}$, $31^{\circ} 30^{\prime} 43^{\prime \prime} \mathrm{N}, 77^{\circ} 48 \quad$ '1"E, 14.iv.18, coll: J.Sengupta, $4 ㅇ ㅜ ㅇ, 5 \delta^{\lambda}$, Sarahan village side, Kinnaur district, $2120 \mathrm{mt}, 31^{\circ} 30^{\prime} 44^{\prime \prime} \mathrm{N}, 77^{\circ} 48$ '1"E 14.iv.18, coll: J.Sengupta.

Diagnosis: Eyes weakly hairy, but hairs can be sparse and inconspicuous, ocellar triangle broadest at base. $3^{\text {rd }}$ and $4^{\text {th }}$ abdominal tergites usually display moustache bands. Tergites carry lunule spots. Front and mid femora with long black posterior hairs in apical quarter. Second basal cell of wing entirely covered in microtrichia. Wing length 8.5-11.75 $\mathrm{mm}$

Distribution: India: Himachal Pradesh, Jammu \& Kashmir, Sikkim, Uttarakhand, West Bengal.

Distribution: elsewhere: Nearctic Region (Alaska, Green land, North Carolina, New Mexico), Oriental Region (Nepal, Pakistan).

Remarks: This species has shown wide distribution throughout Nearctic as well as Oriental region.

V. Genus Chrysotoxum Meigen, 1800

Type species: Musca bicincta Linnaeus.
Diagnosis: Antennae elongated, sometimes longer than head, scape and pedicel often longer than wide. First flagellomere at least 3 times longer. Abdomen convex dorsally, marginated, postero lateral angles of abdominal tergites remain projecting.

\section{Chrysotoxum violaceum Brunetti, $1923^{\star \star}$}

1923. Chrysotoxum violaceum Brunetti, Fauna. Br. India.3:302

Type locality: Darjeeling, India.

Material examined: 19 , Shong Thong, Kinnaur district, $1910 \quad \mathrm{mt}, 31^{\circ} 31^{\prime} 8 \mathrm{~N}, \quad 78^{\circ} 16^{\prime} 13^{\prime \prime} \mathrm{E}$, 13.iv.18, coll: J.Sengupta, $4 \widehat{\jmath}$, Recong Peo, Kinnaur district, $4044 \mathrm{mt}, 32^{\circ} 35^{\prime} 28.59^{\prime \prime} \mathrm{N}$, 76²0'30.94"E,14.iv.18, coll: J.Sengupta,

Diagnosis: Face shining bright yellow in colour, face with median black stripe. Vertex with large violet black spot. Abdominal tergites deep violet blue in colour, scutellum with bright lemon yellow in colour. Legs orange yellow in colour, hind femora wholly black, wing color gradually fading towards grey on hind margin.

Distribution: India: Himachal Pradesh, West Bengal.

Distribution: elsewhere: NIL

Remarks: This species has shown endemic distribution to India, as well as this species has been reported from the first time from this cold and dry zone as well as from the state of Himachal Pradesh.

\section{Tribe Bacchini}

Diagnosis: Face entirely black, abdomen usually slender in shape.

VI. Genus Melanostoma Schiner, 1860

Type species: Musca mellina Linnaeus

Diagnosis: Face entirely black with two distinct central bump, facial pruinescence neither punctated nor rippled. Thorax and scutellum entirely black. Legs in male lacks hairs/hair tufts or bristles.

\section{Melanostoma orientale (Wiedemann, 1824)}

1824. Syrphus orientalis, Wiedemann, Analec. Ent. : 36 
Type locality: Ind. Or

Material examined: 1을 Bhowen, Chamba district, $2121 \mathrm{mt}, 31^{\circ} 31$ '8"N, 7747'46"E,

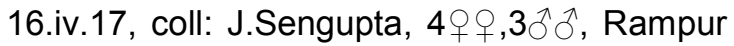
power project, Kinnaur district, $970 \mathrm{mt}$, $31^{\circ} 23^{\prime} 38^{\prime \prime} \mathrm{N}, \quad 77^{\circ} 36^{\prime} 2 " \mathrm{E}, \quad$ 14.iv.18, coll: J.Sengupta, 19, Wangtoo, Kinnaur district, $1580 \mathrm{mt}, 31^{\circ} 33^{\prime} 48 " \mathrm{~N} \quad, \quad 77^{\circ} 59 \quad$ '30"E, 14.iv.18, coll: J.Sengupta, $1 \delta^{\lambda}$, Peo hill side, Kinnaur district, $2000 \mathrm{mt}, 31^{\circ} 29^{\prime} 58^{\prime \prime} \mathrm{N}, 78^{\circ} 13$ '27"E, 14.iv.18, coll: J.Sengupta, $2 \hat{\delta} \partial^{\lambda}$, Recong Peo, Kinnaur district, $4044 \mathrm{mt}, 32^{\circ} 35^{\prime 28.59 " N}$,

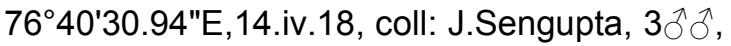
Indrasen, Lahul \& Spiti district, $4134 \mathrm{mt}$, $32^{\circ} 15^{\prime} 52.47^{\prime \prime} \mathrm{N}, 77^{\circ} 29^{\prime} 1.18 \mathrm{E}, \quad 22 . i v .18, \quad$ coll: J.Sengupta.

Diagnosis: Blackish antennae with microscopic pubescent arista, face with small two bump like structure, $3^{\text {rd }}$ and $4^{\text {th }}$ abdominal tergites with a pair of big quadrate to oblong orange yellow spots. Hind tibiae with broad black median stripe. Wings with normal syrphid venation.

Distribution: India: Assam, Arunachal Pradesh, Himachal Pradesh, Jammu \& Kashmir, Karnataka, Meghalaya, Sikkim, Tamil Nadu, Tripura, Uttar Pradesh, and West Bengal.

Distribution: elsewhere: Oriental Region (Bhutan, Nepal, Pakistan, Sri Lanka)

Remarks: Apparently the commonest and most widely distributed species of the genus in the East, occurring throughout the warm weather in the plains and hills.

Tribe Paragini

Diagnosis: Species usually very small, third antennal segment modestly elongated.

VII. Genus Paragus Latreille, 1804 Type species: Mulio bicolor Fabricius.

Diagnosis: Eyes distinctly haired, $1^{\text {st }}$ abdominal tergites well developed, tergites always minutely punctated.

\section{> Subgenus Paragus Latreille, 1804}

\section{Paragus (Paragus) bicolor (Fabricius, 1794)}

1794. Syrphus bicolor Fabricius, Ent. Syst. aucta. 4(6):297
1865. Paragus tacchettii Rondani, Atti. Soc. Ital. Sci. Nat. Mus. Civ. Nat. Milano.8:140

\section{Type locality: Barbariae}

Material examined: $3 q q$, Tapri garden side, Kinnaur district, $1600 \mathrm{mt}, 31^{\circ} 32^{\prime} 5 " \mathrm{~N}, 78^{\circ} 1$ '8"E, 13.iv.18, coll: J.Sengupta,

Diagnosis: $3^{\text {rd }}$ antennal joint noticeably elongated, dark thorax with indistinct pale lines on disc, frons usually shinning blue black in colour, $2^{\text {nd }}$ and $3^{\text {rd }}$ abdominal tergites with reddish secluded spots. Hind metatarsi marginally puffy, clear wing with brownish stigma.

Distribution: India: Himachal Pradesh, Jammu \& Kashmir.

Distribution: elsewhere: Oriental Region (Afghanistan; Pakistan), Palearctic Region (Austria, France, Italy).

Remarks: Larvae of this species feed on aphids occurring on Centaurea and Sonchus.

\section{Subfamily Eristalinae}

Diagnosis: Anterior cross vein at or after middle of discal cell, marginal cell always remain closed. $3^{\text {rd }}$ vein diagonally looped downward into first posterior cell.

Tribe Volucellini

Diagnosis: Upper outer cross vein re-entrant, arista plumose.

VIII. Genus Volucella Geoffroy, 1762

Type species: Musca bombylans Linnaeus.

Diagnosis: Long haired flies. Apical portion of $M_{1}$ curved, strongly toward wing base, anterior anepsternum haired. This genera is well known for mimicking bumble bees.

\section{Volucella ruficauda Brunetti, 1907}

1907. Volucella ruficauda Brunetti, Rec. Indian Mus.1: 379

Type locality: Sikkim, India.

Material examined: $2+\%$, Choling garden, Kinnaur district, $1780 \mathrm{mt}, 31^{\circ} 30^{\prime} 60^{\prime \prime} \mathrm{N}, 78^{\circ} 9$ '9"E,13.iv.18, coll: J.Sengupta, 1+, Tapri garden side, Kinnaur district, 1600 $\mathrm{mt}, 31^{\circ} 32^{\prime} 5 " \mathrm{~N}, \quad 78^{\circ} 1$ '8"E, 13.iv.18, coll: J.Sengupta, $2 \circ+$, Shong Thong, Kinnaur 
district,1910 mt,31'31'8"N, $\quad 78^{\circ} 16^{\prime} 13^{\prime \prime} \mathrm{E}$, 13.iv.18, coll: J.Sengupta.

Diagnosis: Well shaped epistome with ferruginous antennae. Scutellum is also bright ferruginous red in colour. Legs slender and wholly dark in colour. Large brown uneven spot in the centre of the forefront border of wing.

Distribution: India: Himachal Pradesh, Jammu \& Kashmir, Sikkim.

Distribution: elsewhere: NIL

Remarks: This species shows batesian mimicry with bumble bees. This species has shown endemic distribution to India.

IX. Genus Sphiximorpha Rondani, 1850

Type species: Ceria subsessilis Illiger

Diagnosis: First flagellomere shorter than both scape and pedicel, frontal prominence mostly absent, post coxal bridge incomplete. Bases of hind coxae consist of a membranous area on the above surface.

\section{Sphiximorpha triangulifera (Brunetti, 1913) $)^{\star \star}$}

913. Ceria triangulifera Brunetti, Rec. Indian Mus.9: 273

Type locality: Darjeeling District, India

Material examined: 1, Bhowen, Chamba district, $2121 \mathrm{mt}, \quad 31^{\circ} 31^{\prime} 8^{\prime \prime} \mathrm{N}, \quad 77^{\circ} 47^{\prime} 46^{\prime \prime} \mathrm{E}$, 16.iv.17, coll: J.Sengupta.

Diagnosis: Large semi-circular black spot present embracing the antennal prominence, which is ferruginous brown in colour. Yellow scutellum with a basal black crescent spot. $1^{\text {st }}$ abdominal tergites with yellow spots at each side, hind pair of legs with a transitional black band which is extensively wider on under side. Spurious vein narrowly but very distinctly infuscated.

Distribution: India: Himachal Pradesh, West Bengal

Distribution: elsewhere: NIL

Remarks: This species usually show several intra species variation especially in the leg colour. Besides this species has been reported from the first time from this cold and dry zone as well as from the state of Himachal Pradesh.
This species has also shown endemic distribution to India.

Tribe Eristalini

Diagnosis: Wing with $\mathrm{R}_{4+5}$ strongly looped, lower and upper outer cross veins form an almost continuous vein parallel with wing margin.

X. Genus Eristalinus Rondani, 1845

Type species: Musca sepulchralis Linnaeus

Diagnosis: Distinguished dark spots across eyes, anepimeron below the wing base is partly haired. Tuft of strong black hairs on post alar ridge.

> Subgenus Eristalodes Mik, 1897

\section{Eristalinus (Eristalodes) paria (Bigot, 1880)}

1880. Eristalomyia paria Bigot, Ann. Soc. Ent. Fr. ser. 5, 10: 218

1916. Eristalis arisanus Matsumura, Thousand insects of Japan. Additamenta.2:264

Type locality: Sri Lanka.

Material examined: $3 ㅇ$, Sarahan village side, Kinnaur district, $2020 \mathrm{mt}, 31^{\circ} 31$ '8"N, $77^{\circ} 47^{\prime} 46 " \mathrm{E}$, 14.iv.18, coll: J.Sengupta, 1 \%, Kalpa garden area, Kinnaur district, $2810 \mathrm{mt}$, $31^{\circ} 32^{\prime 2} 24^{\prime \prime N}, \quad 78^{\circ} 15$ '9"E, 14.iv.18, coll: J.Sengupta, $3 \circ \circ, \quad 2 \hat{\jmath} \hat{\jmath}$, Sangla, Kinnaur district, $2650 \mathrm{mt}$, 31'25'35"N,78¹5'52"E,14.iv.18, coll: J.Sengupta.

Diagnosis: Face with 2 bare broad longitudinal median stripes on each side of the central bump. Eyes with six narrow parallel longitudinal dark stripes. Thorax dorsum with four approximately equal dull black stripes, abdominal tergites yellowish with black stripes on dorsum. Legs mainly aeneous black, minute dark brown spot at tip or auxiliary vein.

Distribution: India: Himachal Pradesh, Arunachal Pradesh, Chandigarh, Jammu \& Kashmir Karnataka, Manipur, Meghalaya, Mizoram, Sikkim, Tamil Nadu, Uttarakhand, Uttar Pradesh, West Bengal. 
Distribution: elsewhere: Oriental Region (Sri Lanka, Taiwan), Indo-Australian Region (Java, Moluccas)

Remarks: This species is distinguished by the presence of longitudinal stripes on eye.

XI. Genus Eristalis Latreille, 1804

Type species: Musca tenax Linnaeus

Diagnosis: Katepimeron haired, scutum without patches of yellow tomentum, $3^{\text {rd }}$ vein of wing looped downward into $1^{\text {st }}$ posterior cell.

\section{Subgenus Eoseristalis Kanervo, 1938}

\section{Eristalis (Eoseristalis) cerealis Fabricius, 1805}

1805. Eristalis cerealis Fabricius, Syst. Antliat.14: 232

1880. Eristalis barbata Bigot, Ann. Soc. Ent. Fr. ser. 5, 10:214

Type locality: China.

Material examined: : $4 ㅇ+1,1 \hat{0}$, Hadsar, Chamba district, $3100 \mathrm{mt}, 32^{\circ} 27^{\prime} 22.08^{\prime \prime} \mathrm{N}$, 76³6'53.16"E, 16.iv.17, coll: J.Sengupta,

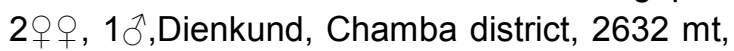
$32^{\circ} 31^{\prime} 7.11^{\prime \prime} \mathrm{N}, \quad 76^{\circ} 2^{\prime} 1.8^{\prime \prime E}, 16 . i v .17$, coll: J.Sengupta, $3 \hat{\delta} \hat{\sigma}, 2+\rho+$, Sarahan village, Kinnaur district, $1820 \mathrm{mt}, 31^{\circ} 31^{\prime} 8 \mathrm{~N} \mathrm{~N}, 77^{\circ} 47$ '30"E, 13.iv.18, coll: J.Sengupta, 2우, Tapri, Kinnaur district, $1760 \mathrm{mt}, \quad 31^{\circ} 30^{\prime} 53^{\prime \prime} \mathrm{N}$, 78 $77^{\prime} 30 " \mathrm{E}, 13 . i v .18$, coll: J.Sengupta, 1, Saltuj, Kinnaur district, $1681 \mathrm{mt}, 31^{\circ} 31^{\prime} \mathrm{O}^{\prime} \mathrm{N}$, $78^{\circ} 5$ '48"E, 13.iv.18, coll: J.Sengupta, $1{ }^{\lambda}$, 2우, Bandhal, Kinnaur district, $1570 \mathrm{mt}$, $31^{\circ} 32 ' 38 " \mathrm{~N}, \quad 77^{\circ} 49$ '15"E, 13.iv.18, coll: J.Sengupta, 1, Recong Peo, Kinnaur district, $2490 \mathrm{mt}, 31^{\circ} 32^{\prime 22} \mathrm{~N}, 78^{\circ} 16$ '11"E, 13.iv.18, coll: J.Sengupta, $4 ㅇ+$, Garam Pani area, Kinnaur district, $1670 \mathrm{mt}, \quad 31^{\circ} 31^{\prime 2} \mathrm{\prime} \mathrm{N}$, 786'19"E, 13.iv.18, coll: J.Sengupta,

Diagnosis: Dark antennae with long feathered arista, frons with dense black or dark brown pubescence, thorax with a soberly wide oblique stripe, $2^{\text {nd }}$ abdominal tergite with a pair of big triangular spot, presence of pale yellow and black hairs blended on black parts of tibia and tarsi. Wing with $3^{\text {rd }}$ vein looped downward into $1^{\text {st }}$ posterior cell.
Distribution: India: Assam, Himachal Pradesh, Jammu \& Kashmir, Meghalaya, Sikkim, Tamil Nadu, West Bengal.

Distribution: elsewhere: Widespread in Oriental region, Palearctic region (Russia)

Remarks: This species looks quite similar with tenax, apparently common throughout the Himalayas throughout the summer.

\section{Eristalis (Eoseristalis) himalayensis Brunetti, 1908}

1908. Eristalis himalayensis Brunetti,Rec. Indian Mus.2:70

Type locality: Indostan

Material examined: $1 \hat{\delta}$, Chitkul village, Kinnaur district, $3140 \mathrm{mt}, 31^{\circ} 22^{\prime} 45^{\prime \prime} \mathrm{N} \quad$,78²2'6"E, 13.iv.18, coll: J.Sengupta, $2 q$, , Chitkul bridge side, Kinnaur district, $3420 \mathrm{mt}$, $31^{\circ} 21^{\prime} 1 " \mathrm{~N}, 78^{\circ} 26^{\prime} 10^{\prime \prime} \mathrm{E}, \quad$ 13.iv.18, coll: J.Sengupta,

Diagnosis: Eyes wholly covered with pubescence, additionally band of dense dark brown pubescence present. Central bump less prominent. Blackish antennae with strongly plumose brownish arista. Thorax blackish with bright yellow scutellum. Abdomen bluntly conical, blackish. Legs completely black. A large yellow brown spot present at middle of costa.

Distribution: India: Himachal Pradesh, Arunachal Pradesh, Jammu \& Kashmir, Sikkim, Uttarakhand, West Bengal

Distribution: elsewhere: Oriental Region (China, Java, Myanmar, Nepal Sri Lanka). Indo-Australian Region (Malaya, Philippines, Sumatra, Sumbawa.)

Remarks: This species is apparently common throughout the Himalayas above the elevational range of 5,000 feet throughout the summer.

Subgenus Eristalis Latreille, 1804

\section{Eristalis (Eristalis) tenax (Linnaeus, 1758)}

1758. Musca tenax Linnaeus, Syst. Nat. Ed. 10: 591

1924. Eristalis claripes Abreu, Mems R. Acad. Cienc. Artes. 19(1): 104 
Type locality: Europe

Material examined: $7 \hat{\delta} \widehat{\delta}, 4+q$, Dienkund, Chamba district, $2632 \mathrm{mt}, 32^{\circ} 31^{\prime} 7.11^{\prime \prime} \mathrm{N}$, 76²'1.8"E, 15.iv.17, coll: J.Sengupta, $2 \widehat{ }{ }^{\circ}$, 6우으. Hadsar, Chamba district, $3100 \mathrm{mt}$, $32^{\circ} 27^{\prime} 22.08^{\prime \prime} \mathrm{N}, 76^{\circ} 36^{\prime} 53.16 " \mathrm{E}, 16 . \mathrm{iv} .17$, coll: J.Sengupta, $2 \hat{\partial}$, Sarahan village, Kinnaur district, $1960 \mathrm{mt}, 31^{\circ} 30^{\prime} 58 \mathrm{~N}^{\mathrm{N}} \mathrm{N}, 77^{\circ} 47$ '50"E, 13.iv.18, coll: J.Sengupta, $2 \hat{\partial} \hat{\jmath}, 2$ q $q$ Sarahan village, Kinnaur district, $2000 \mathrm{mt}$,

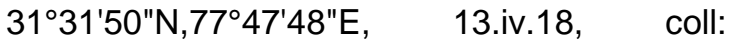
J.Sengupta, 1 ㅇ Sarahan village panchayet area, Kinnaur district, $1710 \mathrm{mt}$,

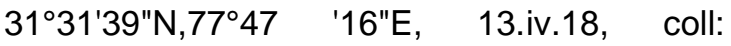
J.Sengupta, 390 hill side road, Kinnaur, Kinnaur district, $1600 \mathrm{mt}$,

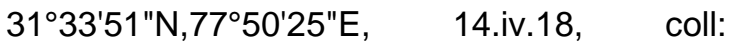
J.Sengupta, $2 \delta^{\lambda}, 1+$ Chowra, Kinnaur district, $1540 \mathrm{mt}, 31^{\circ} 34^{\prime} 28^{\prime \prime} \mathrm{N}, 78^{\circ} 51^{\prime} 14 " \mathrm{E}, 14 . i v .18$, coll: J.Sengupta, $1 \delta^{\lambda}, 1$ 우 Salding, Kinnaur district, $\quad 1600 \quad \mathrm{mt}, \quad 31^{\circ} 33^{\prime} 46^{\prime \prime} \mathrm{N}$, $78^{\circ} 58^{\prime} 21 " E, 14 . i v .18$, coll: J.Sengupta, $1 \delta^{\top}, 1$ 우 Nathpa, Kinnaur district, $1690 \mathrm{mt}$, 31'30'59"N,785'50"E, 14.iv.18, coll: J.Sengupta, $3 \delta^{\hat{}}$,Wangtoo, Kinnaur district, $1680 \mathrm{mt}, 31^{\circ} 34^{\prime} 20 " \mathrm{~N}, 77^{\circ} 51$ '25"E, 14.iv.18, coll: J.Sengupta, $3 \hat{\jmath} \partial^{\lambda}, 2$ 우 Saltuj river side, Kinnaur district, $1810 \mathrm{mt}$, 31⒉'16"N,78¹0'58"E, 13.iv.18, coll: J.Sengupta, 2 우 Sangla, Kinnaur district, $2520 \mathrm{mt}, 31^{\circ} 26^{\prime} 1 " \mathrm{~N}, 78^{\circ} 14^{\prime} 53^{\prime \prime E}$, 13.iv.18, coll: J.Sengupta, $3 \hat{0}, 40+$ Sangla, Kinnaur district, $3070 \mathrm{mt}, \quad 31^{\circ} 23^{\prime} 50^{\prime \prime N}, 78^{\circ} 20^{\prime} 52^{\prime \prime} \mathrm{E}$, 13.iv.18, coll: J.Sengupta, 1 i Rakcham Valley, Kinnaur district, $2770 \mathrm{mt}$, 31 $25^{\prime} 40^{\prime \prime} \mathrm{N}, 78^{\circ} 15^{\prime} 50 " \mathrm{E}, \quad$ 13.iv.18, coll: J.Sengupta, 29 우 Nogulsari, Kinnaur district, $1650 \mathrm{mt}, 31^{\circ} 33^{\prime} 27^{\prime \prime N}, 77^{\circ} 52$ '59"E, 14.iv.18, coll: J.Sengupta, $2 \hat{\jmath} \hat{\sigma}$, Trinda Mataji Mandir area, Kinnaur district, $1600 \mathrm{mt}$, $31^{\circ} 34^{\prime} 9 " \mathrm{~N}, 77^{\circ} 53 \quad$ '17"E, 13.iv.18, coll: J.Sengupta, 19 Sungra, Kinnaur district, 1530 $\mathrm{mt}, \quad 31^{\circ} 34^{\prime} 4 " \mathrm{~N}, 77^{\circ} 55 \quad$ '46"E,15.iv.18, coll: J.Sengupta, $2 \delta^{\lambda} \sigma^{\lambda}$ Bhaba nagar river dam side, Kinnaur district, $1530 \mathrm{mt}, 31^{\circ} 33^{\prime} 45^{\prime \prime} \mathrm{N}, 7^{\circ} 58$ '41"E,15.iv.18, coll: J.Sengupta, $5 \AA 11$ 우 Tukpa valley, Kinnaur district, $2530 \mathrm{mt}, 31^{\circ} 25^{\prime} 59^{\prime \prime} \mathrm{N}$, 78०14'36"E,15.iv.18, coll: J.Sengupta, 1 ㅇ Tharmanga village, Kinnaur district, $2660 \mathrm{mt}$, 31'25'39"N,78¹5'51"E,15.iv.18, coll: J.Sengupta, $2 \hat{\jmath} \partial^{\hat{2}}$, Batal, Lahul \& Spiti district, $4489 \mathrm{mt}, 32^{\circ} 17^{\prime} 58.04^{\prime \prime} \mathrm{N} 77^{\circ} 36{ }^{\prime} 34.95 " \mathrm{E}$, 19.iv.18, coll: J.Sengupta, $3 \widehat{\jmath}$, Gemur, Lahul \& Spiti district, $4827 \mathrm{mt}, 32^{\circ} 36^{\prime} 21.32^{\prime \prime} \mathrm{N}$, 77010'11.76"E, 20.iv.18,coll: J. Sengupta, $29+$, Kugti, Lahul \& Spiti district, $4630 \mathrm{mt}$, $32^{\circ} 28^{\prime} 42.58^{\prime \prime} \mathrm{N}, 76^{\circ} 53^{\prime} 5.95 " \mathrm{E}, 19 . i v .18$, coll: J.Sengupta,

Diagnosis: Face with black central stripe, very wide. Stripes of dark hairs down the eyes, almost completely dark hind tibia with long hairs on the dorsal and ventral surface and the very broad black face stripe. The abdominal tergites vary in colour range of abdominal markings from black to orange. Wing length 9.75-13 mm.

Distribution: India: Himachal Pradesh, Arunachal Pradesh, Chandigarh, Jammu \& Kashmir, Manipur, Meghalaya, Mizoram, Nagaland, Punjab, Sikkim, Uttarakhand, West Bengal.

Distribution: elsewhere: Australasian Region (Australia), Indo-Australian Region (Hawaii), Palaearctic Region (China, Japan), Oriental Region (Myanmar, Pakistan, Sri Lanka), Australasian Region (New Zealand).

Remarks: This species is reported to cause accidental genital myiasis in human from Iran in the year 2010. (Gonzalez et.al, 2009)

Xenus Phytomia Guerin-Meneville,
1833

Type species: Eristalis chrysopygus Wiedemann

Diagnosis: Whole body densely punctuate, presence of a small wrinkled surface just below the antennae, $3^{\text {rd }}$ antennal joint oblong and ovate, drooping, scutellum distinctly large.

\section{$>$ Subgenus Dolichomerus Macquart, 1850}

\section{Phytomia (Dolichomerus) crassa} (Fabricius, 1787)

1787. Syrphus crassus Fabricius, Mant. Ins. 2:334

1849. Dolichomerus crassus, Macquart, Dipt. Exot., 4: 132

Type locality: Tranquebar, Chennai, Tamil Nadu, India 
Material examined:2 $q 9$ Khokpa, Kinnaur district, $3652 \mathrm{mt}, \quad 31^{\circ} 35^{\prime} 12.81^{\prime \prime} \mathrm{N}$, 78²7'19.17"E, 15.iv.18,coll: J. Sengupta.

Diagnosis: Frontal callus mostly conquer $3 / 4^{\text {th }}$ of frontal triangle, blackish antennae with plumose orange arista, $2^{\text {nd }}$ to $4^{\text {th }}$ abdominal tergites with a deep imprint of a large circle. A slender dark brown stripe running through $2^{\text {nd }}$ to $4^{\text {th }}$ vein encircles the anterior cross vein of the wing.

Distribution: India: Himachal Pradesh, Andhra Pradesh, Assam, Chennai (Tamil Nadu).

Distribution: elsewhere: Oriental region (Sri Lanka, Laos, Nepal, Thailand), Indo-Australian Region (Sulawesi, Malaya)

Remarks: This species is characterised by the presence of toothed a hind femora, a species widely distributed throughout Oriental region.

XIII. Genus Mallota Meigen, 1822

Type species: Syrphus fuciformis Fabricius

Diagnosis: Flies are remarkably long haired, Abdomen dark coloured without any distinguished pale markings. They efficiently mimics bumble bee and other hairy bees.

$>$ Subgenus Mallota Meigen, 1822

\section{Mallota}

(Mallota)

orientalis (Wiedemann, 1824) $^{\star *}$

1842. Imatisma orientalis Macquart, Dipt. Exot. 2(2):69

Type locality: Indonesia. Java

Material examined: $2 q q$, Dhar Mane Rang, Kinnaur district, $4930 \mathrm{mt}, 32^{\circ} 0{ }^{\prime} 43.27 " \mathrm{~N}$ 78¹9'33.83"E,19.iv.18, coll: J.Sengupta.

Diagnosis: Dark antennae with sub basal arista, sub quadrate thorax with relatively small blackish scutellum, $2^{\text {nd }}$ and $3^{\text {rd }}$ abdominal tergites broader than other segments. Incrassate hind legs with a tuft of black hairs below, wing with a brownish spot at base of sub marginal cell.

Distribution: India: Himachal Pradesh, Sikkim, West Bengal.

Distribution: elsewhere: Oriental region (Taiwan, Java, Laos)
Remarks: This species has been reported from the first time from this cold and dry zone as well as from the state of Himachal Pradesh.

\section{Mallota (Mallota) varicolor (Walker, 1856) $)^{\star \star}$}

1857. Merodon varicolor, Walker, Proc. Linn. Soc. Lond. 1: 122

Type locality: Malaysia. Sarawak.

Material examined: 1q, Mahoun, Kinnaur district, $\quad 4304 \mathrm{mt}, \quad 32^{\circ} 23^{\prime} 54.01^{\prime \prime} \mathrm{N}$, 76³9'11.90"E,19.iv.18, coll: J.Sengupta, 3 우오, Sarahan village side, Kinnaur district, $2110 \mathrm{mt}, \quad 31^{\circ} 31^{\prime} 18^{\prime \prime} \mathrm{N}, 77^{\circ} 47$ '52"E, 13.iv.18, coll: J.Sengupta,

Diagnosis: Frons magnificently black, face with a medium dark brown tripe, dorsum of thorax with four equidistant narrow longitudinal stripe, $2^{\text {nd }}$ abdominal tergite with a diamond shaped yellow spots, hind legs with a distinctive tooth just beyond base, a dark brownish stain in stigmatic region.

Distribution: India: Himachal Pradesh, Assam, Meghalaya, West Bengal.

Distribution: elsewhere: Indo- Australian Region (Borneo)

Remarks: This species has been reported from the first time from this cold and dry zone as well as from the state of Himachal Pradesh. According to literature, this species is mainly confined to high altitudinal landscape.

XIV. Genus Syritta Lepeletier \& Serville, 1828

Type species: Musca pipiens Linnaeus.

Diagnosis: Metasternum haired, hairs longer than hind coxa, hind femur distinguishingly greatly enlarged. On apical third an anteroventral spinose ridge is present.

18. Syritta pipiens (Linnaeus, 1758)

1758. Musca pipiens Linnaeus, Systema naturae.1: 594

1974. Spheginoides tenofemorus Dzhafarova, Uchen. Zap. Univ., Ser. biol. Baku.1: 40

Type locality: Europe 
Material examined: $3 \hat{\widehat{\partial}} \hat{,}$ 5우 Dhar Ula, Kinnaur district, $4710 \mathrm{mt}, 32^{\circ} 6^{\prime} 52.73^{\prime \prime} \mathrm{N}$, $77^{\circ} 58^{\prime} 45.43^{\prime \prime E}, 16 . i v .18$,coll: J. Sengupta, 2 우 Dhar Sajam, Kinnaur district, $4975 \mathrm{mt}$, $32^{\circ} 54^{\prime} 8.73 " \mathrm{~N}, 77^{\circ} 40^{\prime} 37.98 " \mathrm{E}, 16 . i v .18$,coll: J. Sengupta, $4+q$ Sarahan, Kinnaur district, 1280 $\mathrm{mt}$, 31 $30^{\circ} 53^{\prime \prime} \mathrm{N}, 7^{\circ} 44^{\prime} 56 " \mathrm{E}, 13 . i v .18$,coll: J. Sengupta, $2 \widehat{\jmath}, 1+$ Sangra, Kinnaur district, $1450 \mathrm{mt}, 31^{\circ} 33^{\prime} 16^{\prime \prime} \mathrm{N}, 77^{\circ} 55$ '7"E, 13.iv.18,coll: J. Sengupta, $1 q$ Kuppa, Kinnaur district, 2070 $\mathrm{mt}, 31^{\circ} 28^{\prime} 13^{\prime \prime N}, \quad 78^{\circ} 11^{\prime} 9 " \mathrm{E}, 13 . i v .18$,coll: J. Sengupta.

Diagnosis: Small narrow fly. Hind femur swollen in a compact manner, with small spines beneath apically. Presence of long spine at the base of inner surface of hind femur. Wing length 4.25-7 mm.

Distribution: India: Himachal Pradesh, Jammu \& Kashmir, Uttarakhand, Uttar Pradesh and West Bengal.

Distribution: Nearctic Region (California \& Florida), Neotropical region (British Columbia, Mexico)

Remarks: This species is distinguished by the highly swollen area of hind leg.

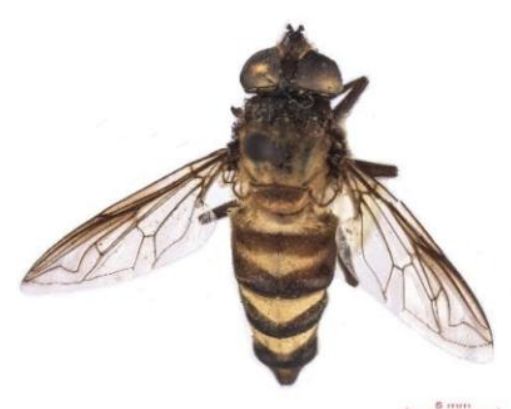

$3 \mathrm{~A}$

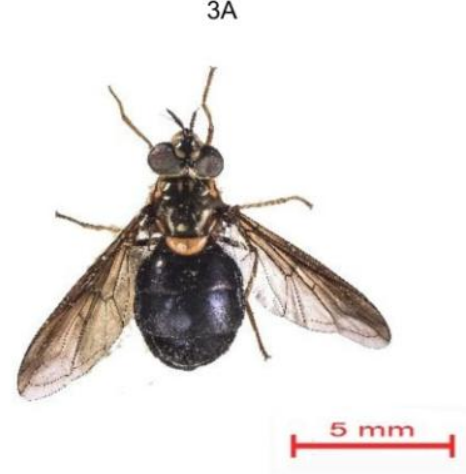

3D

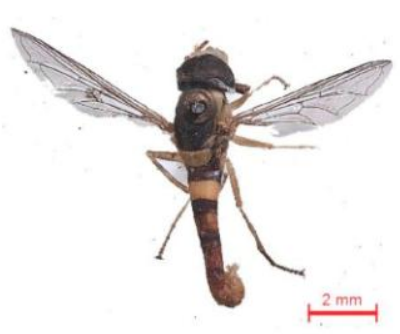

$3 G$

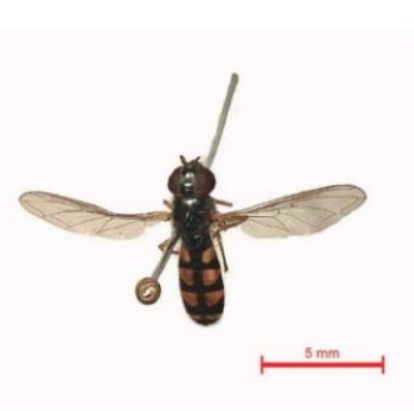

$3 B$

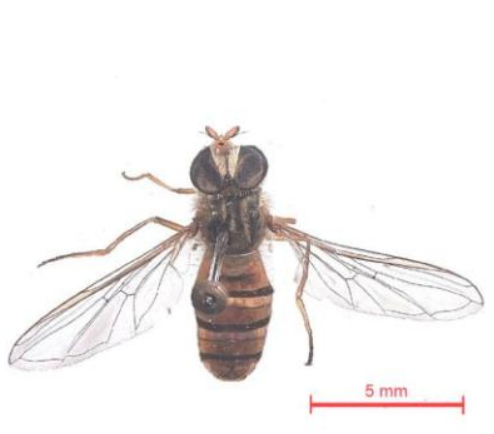

3E

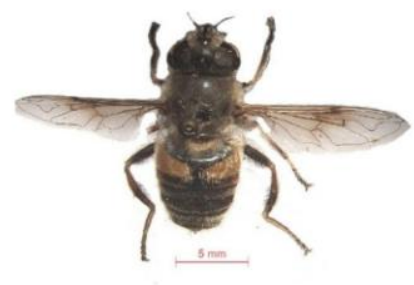

$3 \mathrm{H}$

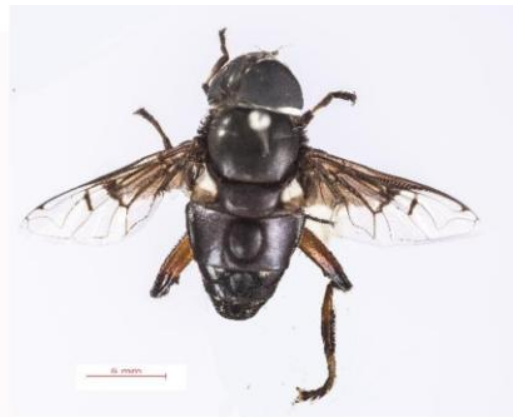

$3 C$

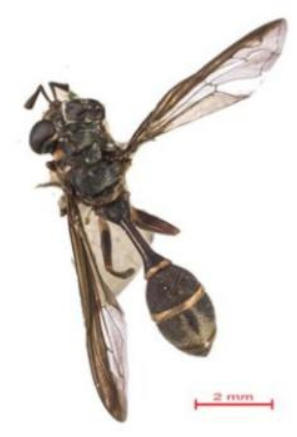

$3 F$

Figure 3A-3I: Habitus of 3A: Mallota (Mallota) varicolor (Walker, 1856),3B: Melanostoma orientale (Wiedemann, 1824), 3C: Phytomia (Dolichomerus) crassa (Fabricius, 1787), 3D: Chrysotoxum violaceum Brunetti, 1923, 3E:Episyrphus (Episyrphus) balteatus (De Geer, 1776), 3F:Sphiximorpha triangulifera (Brunetti, 1913),3G:Sphaerophoria (Sphaerophoria) indiana Bigot, 1884, 3H:Eristalis (Eristalis) tenax (Linnaeus, 1758), 3l:Volucella ruficauda Brunetti, 1907. 


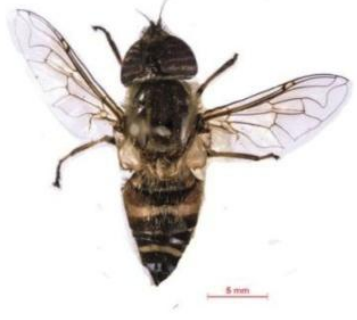

$4 \mathrm{~A}$

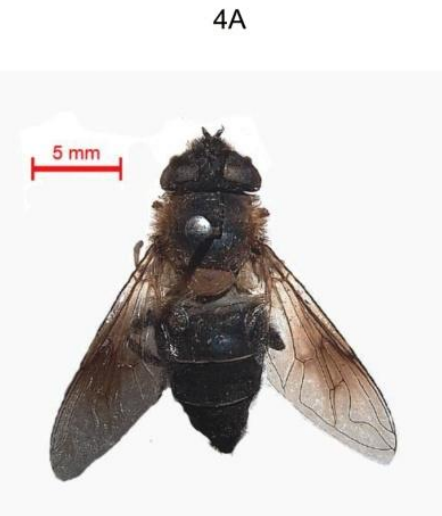

$4 \mathrm{D}$

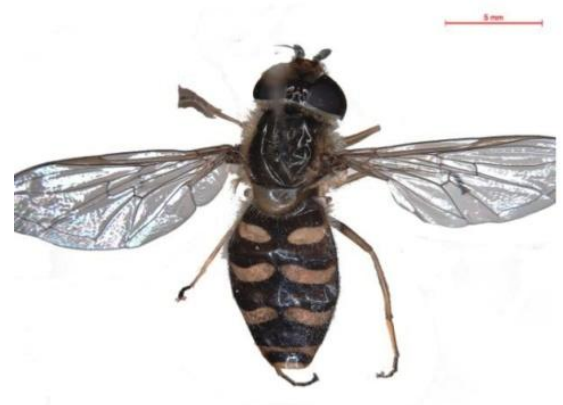

$4 G$

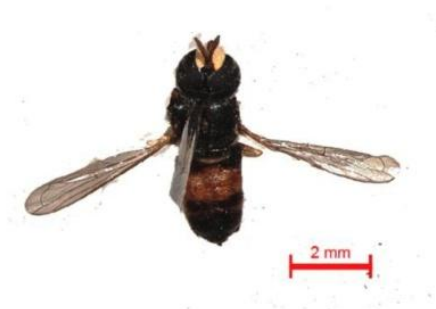

4B

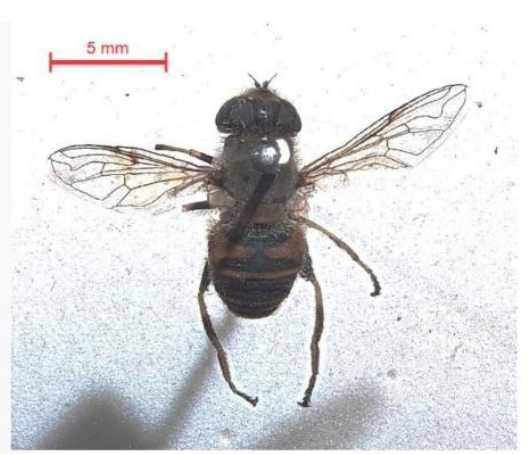

$4 \mathrm{E}$

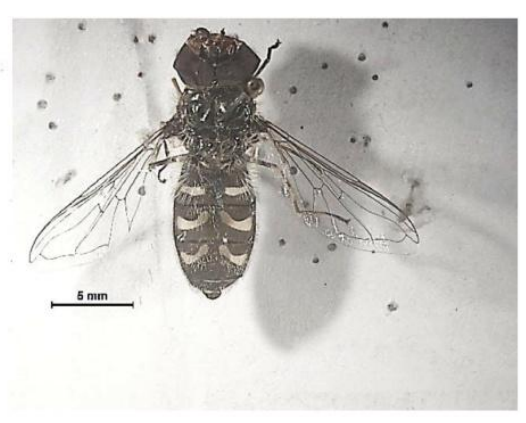

$4 C$

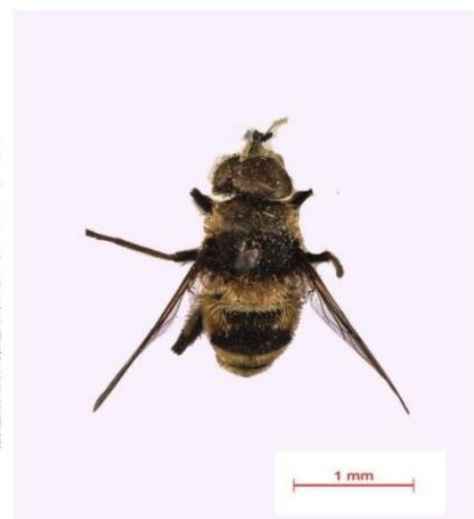

$4 \mathrm{~F}$

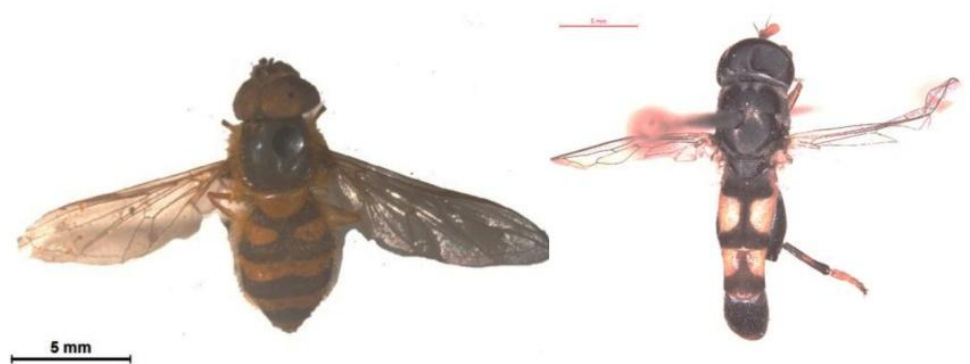

$4 \mathrm{H}$

41

Figure 4A-4I: Habitus of 4A: Eristalinus (Eristalodes) paria (Bigot, 1880),4B: Paragus (Paragus) bicolor (Fabricius, 1794), 4C: Scaeva pyrastri (Linnaeus, 1758), 4D: Eristalis (Eoseristalis) himalayensis Brunetti, 1908, 4E: Eristalis (Eoseristalis) cerealis Fabricius, 1805, 4F: Mallota (Mallota) orientalis (Wiedemann, 1824), 4G: Scaeva latimaculata (Brunetti, 1923), 4H: Syrphus (Syrphus) torvus Osten Sacken, 1875, 4I:Syritta pipiens (Linnaeus, 1758)

\section{Conclusion}

Altogether 18 species of hoverflies under 14 genera and 2 sub families have been reported from our study area. Among which 4 species namely Chrysotoxum violaceum Brunetti, 1923, Sphiximorpha triangulifera (Brunetti, 1913), Mallota (Mallota) orientalis (Wiedemann, 1824), Mallota (Mallota) varicolor (Walker, 1856) has been reported from the first time from this cold dry zone as

well as from the state of Himachal Pradesh. Hoverflies are found to be abundant mostly during late morning to early noon time throughout all the season, although during winter season this zone of Himachal Pradesh remain isolated from rest of the world due to very heavy snow fall, thus abundance of hoverflies during winter season could not be determined from this zone. This is one of the 
gap areas which should be attempted in future days. Among the reported 18 species, 6 species found to be widely distributed throughout the year while 3 species found to be endemic from the state as well as from India. As this zone of Himachal Pradesh is very close to the boundary of China and Tibet, so many of the species reported from this area has shown a more oriented distribution towards the Palaearctic region. But overall the species richness as well as species abundance is low from this area which is mostly due to unfavourable climatic condition excessive low level of precipitation as well as adverse geographical characteristics including dry and cold landscape of this zone. Further

\section{References}

Ankersmit, G. W., Dijkman, H., Keuning, N. J., Mertens, H., Sins, A., \& Tacoma, H. M. (1986). Episyrphus balteatus as a predator of the aphid Sitobion avenae on winter wheat. Entomologia experimentalis et applicata, 42(3), 271-277.

Brunetti, E. (1907). Notes on the Oriental Syrphidae. Part I. Records of the Indian Museum, 1, 11-13.

Brunetti, E. (1908). Notes on Oriental Syrphidae with descriptions of new species. Part I. Records of the Indian Museum, 2, 4996.

Brunetti, E. (1913). New and interesting Diptera from the eastern Himalayas. Records of the Indian Museum, 9(5), 255-277.

Brunetti, E. (1923). Family Syrphidae. Diptera Brachycera.. (Vol. 3). Taylor \& Francis.

Brunetti, E. (1925). Some notes on Indian Syrphidae, Conopidae and Oestridae. Records of the Indian Museum, 27, 75-79.

Bulganin, M., Parui, P., Mukherjee, M., Sharma, R. M., \& Mehta, H. S. (2003). Flower flies from the Pin Valley National Park, Himachal Pradesh. Bionotes, 5(4).

Ghahari, H., Hayat, R., Tabari, M., \& Ostovan, H. (2008). Hover flies (Diptera: Syrphidae) from rice fields and around grasslands of northern Iran. Munis entomology and zoology, 3(1), 275-284. study from this area especially in winter time will give a clearer picture of hoverfly diversity from this area of Himachal Pradesh.

\section{Acknowledgement}

We wish to express our thanks to Dr. Kailash Chandra, Director, Zoological Survey of India for providing the permission and necessary facilities. Thanks are due to Sri K.C. Gopi, Additional Director, Dr. C. Raghunathan, Divisional. In Charge, Entomology. Division. B, for their continuous encouragement. Further, we acknowledge and convey our sincere thanks to our fellow team members for their constant encouragement.

Ghorpadé, K. (2009). Some nomenclatural notes on Indian subregion Syrphini (Diptera: Syrphidae). Colemania, 15, 3-13.

Ghorpadé, K., \& Shehzad, A. (2013). An annotated checklist and select bibliography of the Hoverflies (Diptera-Syrphidae) of Pakistan, Indian subcontinent. Colemania, 37, 1-26.

Graham E.Rotheray and Francis Gilbert: The Natural History of HOVERFLIES. First edition, Forrest Text Publisher, Iver (United Kingdom), 2011, 334.

Mani, M. S. (2013). Ecology and biogeography of high altitude insects (Vol. 4). Springer Science \& Business Media.

Miranda, G. F. G., Young, A. D., Locke, M. M., Marshall, S. A., Skevington, J. H., \& Thompson, F. C. (2013). Key to the genera of Nearctic Syrphidae. Canadian Journal of Arthropod Identification, 23(1), 351. https://doi.org/10.3752/cjai.2013.23

Mitra, B., Roy, S., Imam, I., \& Ghosh, M. (2015). A review of the hover flies (Syrphidae: Diptera) from India. Int J Fauna Biol Stud, 2, 61-73.

Potts, S. G., Petanidou, T., Roberts, S., O'Toole, C., Hulbert, A., \& Willmer, P. (2006). Plant-pollinator biodiversity and pollination services in a complex Mediterranean 
landscape. Biological conservation, 129(4), 519-529.

https://doi.org/10.1016/j.biocon.2005.11.019

Sengupta, J., Naskar, A., Maity, A., Hazra, S., Mukhopadhyay, E., Banerjee, D., \& Ghosh, S. (2016). An updated distributional account of Indian hover flies (Insecta: Diptera: Syrphidae). J Entomol Zool Stud, 4, 381-396.

Sengupta, J., Naskar, A., Maity, A., Hazra, S., \& Banerjee, D. (2016). NEW DISTRIBUTIONAL RECORDS AND ANNOTATED KEYS OF HOVER FLIES (INSECTA: DIPTERA: SYRPHIDAE) FROM HIMACHAL PRADESH, INDIA. J. Adv. Zool, 37(1), 31-54.

Shah, G. M., Jan, U., \& Wachkoo, A. A. (2014). A checklist of hoverflies (Diptera: Syrphidae) in the western Himalaya, India. Acta Zoologica Hungarica, 60(4), 283305.

Ssymank, A., Kearns, C. A., Pape, T., \& Thompson, F. C. (2008). Pollinating flies (Diptera): a major contribution to plant diversity and agricultural production. Biodiversity, 9(12), 86-89.

https://doi.org/10.1080/14888386.2008.97128 92

Teskey, H.J., Vockeroth, J.R. \& Wood, D.M., (1981). Manual of Nearctic Diptera. Ottawa, Research Branch, Agriculture Canada, Monograph, 27.

Thompson, F. C., \& Pape, T. (2018). Syrphidae. Systema Dipterorum, Version 1.5. 13354 records.
Tinkeu, L. N., \& Hance, T. (1998). Functional morphology of the mandibles of the larvae of Episyrphus balteatus (De Geer, 1776)(Diptera: Syrphidae). International Journal of Insect Morphology and Embryology, 27(2), 135-142. https://doi.org/10.1016/S0020-7322(97)00015$\underline{9}$

Thompson, F. C., Vockeroth, J. R., \& Speight, M. C. (1982). The Linnaean species of flower flies (Diptera: Syrphidae).

Vockeroth, J. R. (1969). A revision of the genera of the Syrphini (Diptera: Syrphidae). The Memoirs of the Entomological Society of Canada, 101(S62), 5-176.

Vockeroth, J. R. (1971). The identity of some holarctic and Old World species of Sphaerophoria (Diptera: Syrphidae). The Canadian Entomologist, 103(11), 1627-1635.

Vockeroth, J. R. (1992). The flower flies of the subfamily Syrphinae of Canada, Alaska, and Greenland: Diptera, Syrphidae (Vol. 1867). Agriculture Canada.

http://publications.gc.ca/pub?id=9.811395\&sl= $\underline{0}$

Vockeroth, J. R., Thompson, F. C., McAlpine, J. F., Peterson, B. V., Shewell, G. E., Teskey, H. J., \& Wood, D. M. (1987). Syrphidae, pp. 713-743. Manual of Nearctic Diptera, 2. http://www.publications.gc.ca/pub?id=9.61031 $\underline{5 \& s l=0}$ 